\title{
The Guidance of Axons from Transplanted Neurons Through Aneural Drosophila Wings
}

\author{
Seth S. Blair, Marjorie A. Murray, and John Palka \\ Department of Zoology, University of Washington, Seattle, Washington 98195
}

\begin{abstract}
The sensory neurons of the wing of Drosophila arise during the first $\mathbf{2 4} \mathrm{hr}$ of metamorphosis, and their axons converge to form a stereotyped set of nerves projecting proximally from the peripherally located cell bodies through the wing and towards the CNS. To better characterize the cues guiding this stereotyped axon outgrowth, we have performed a series of transplantation studies in which neurons from a variety of sources (wing, eye, antenna, and leg disks) were placed into mutant, aneural wings. Axons growing from such implants in effect assay the host wing for the presence and location of guidance cues. Our results show, first, that such axons prefer to grow in the normal, proximal direction and, second, that they prefer to grow along the approximate site of one of the normal nerves, that of the third longitudinal vein. It therefore appears that the aneural wing epithelium contains cues capable of directing both the polarity and the location of axonal outgrowth. These cues are relatively nonspecific, in that a variety of neuronal types are capable of responding to them.
\end{abstract}

The first axons that link the CNS and peripheral structures follow regular trajectories through previously uninnervated territory. For vertebrates, the cues responsible for axon guidance have often been thought of in terms of spatially distinct "highways," pathways whose nature is unknown but whose presence within the peripheral tissues is recognized by the fact that axons from a diversity of sources grow preferentially along them (Summerbell and Stirling, 1982; Landmesser, 1984; Purves and Lichtman, 1985). For insects, 2 other classes of hypothetical guidance cues have frequently been proposed: generalized proximodistal gradients of adhesivity, which are believed to draw axons from their peripherally located cell bodies towards the base of an appendage (Nardi, 1983; Caudy and Bentley, 1986a), and critically positioned structures such as guidepost neurons, which seem to be responsible for bends and other special features in the path (Bentley and Keshishian, 1982; Ho and Goodman, 1982; Bentley and Caudy, 1983a, b; Berlot and Goodman, 1984; Caudy and Bentley, 1986b). We now offer evidence that insects can also have highways in the vertebrate sense: Axons of neurons implanted surgically into mutant, aneural wings of Drosophila grow towards and along a specific path normally followed by axons in wild-type wings. As in vertebrates, this non-neural path

Received Feb. 24, 1987; revised May 26, 1987; accepted June 3, 1987.

We thank R. Sugg for help with sectioning and M. Schubiger for comments and criticism. This research was carried out during the tenure of a Postdoctoral Fellowship from the Muscular Dystrcyhy Association (to S.S.B.) and was supported by NIH Jacob Javits Award NS 07778 and NSF Research Grant BNS 8507460 (to J.P.).

Correspondence should be addressed to Seth S. Blair at the above address.

Copyright (C) 1987 Society for Neuroscience 0270-6474/87/124165-11\$02.00/0 guides axons originating in diverse locations and derived from a variety of neuronal types. Moreover, it can be followed not only in the normal direction, but also in the reverse direction.

The wing of the fruitfly Drosophila melanogaster develops from the wing imaginal disk, an epithelial sack that everts and flattens during metamorphosis to form the wing blade, hinge, and thorax (Waddington, 1940). The sensory neurons of the wing, associated with the campaniform and bristle sensilla, become recognizable and initiate axonogenesis within the wing during the first few hours of metamorphosis (Murray et al., 1984; Jan et al., 1985). By $24 \mathrm{hr}$ after pupariation (AP), the complete complement of neurons has appeared and the mature nerve pattern within the wing has been formed (see Figs. $1 B, 2 A$ ). This pattern consists of 2 nerve bundles that project proximally down the first and third longitudinal veins (L1 and L3) and meet at the junction of these veins to form the major nerve of the radius. This normal pattern of development is preserved when wing disks are explanted and reared in vitro, which makes possible a range of surgical manipulations on the developing wing (Blair and Palka, 1985a; Blair et al., 1985). Several types of possible cues-guidepost neurons (Blair and Palka, 1985a; Schubiger and Palka, 1985), physical channels (Blair et al., 1985), one expression of epithelial polarity (Schubiger and Palka, 1986)-have all been shown to be unnecessary for normal patterned outgrowth in the wing. We have therefore conducted a series of transplantation experiments to test directly the ability of the wing epithelium to guide growing axons (Blair and Palka, 1985b; Blair et al., 1986).

\section{Materials and Methods}

The transplantation procedure and all culturing were performed in Shields and Sang M3 medium (Shields and Sang, 1977) as described previously (Blair et al., 1985). To perform the transplantation (Fig. 1), a small fragment of neuron-containing donor tissue was placed on the tip of a tungsten needle, which was then inserted through the opening between the dorsal and ventral epithelia of a host wing taken at $2 \mathrm{hr}$ AP. The donor tissue was lodged in place by puncturing the host epithelium at the distal tip with the end of the needle, a procedure that should not damage tissue except at the distal tip. The path of the needle and the anteroposterior position of the puncture were deliberately varied from wing to wing. To further rule out the path of the needle as a possible guide for axons, the donor tissue was in some experiments inserted into the host wing directly through the dorsal or ventral epithelium at right angles to the proximodistal axis. Operated wings were reared for approximately $24 \mathrm{hr}$ in vitro and then fixed, stained with anti-HRP and an FITC-conjugated secondary antiserum, and mounted as described previously (Blair et al., 1985). Wings for sectioning were stained using anti-HRP and the ABC Vectastain system, examined in whole mount, and then embedded, sectioned, and counterstained as described previously (Blair et al., 1985). At least 3 wings of each type (see Results) were sectioned and examined.

Aneural host wings were picked from 2 stocks: $y \mathrm{Hw} / \mathrm{sc}^{\prime 0-1}$ (Caltech) and $y H w \beta 36 / s^{10-1} \beta^{36 a}$ (Madrid). The $s c^{10-1} \beta^{36 a}$ wings contained immunoreactive debris, which sometimes lay in the wing channels $(12 \mathrm{hr}$ 
DONOR

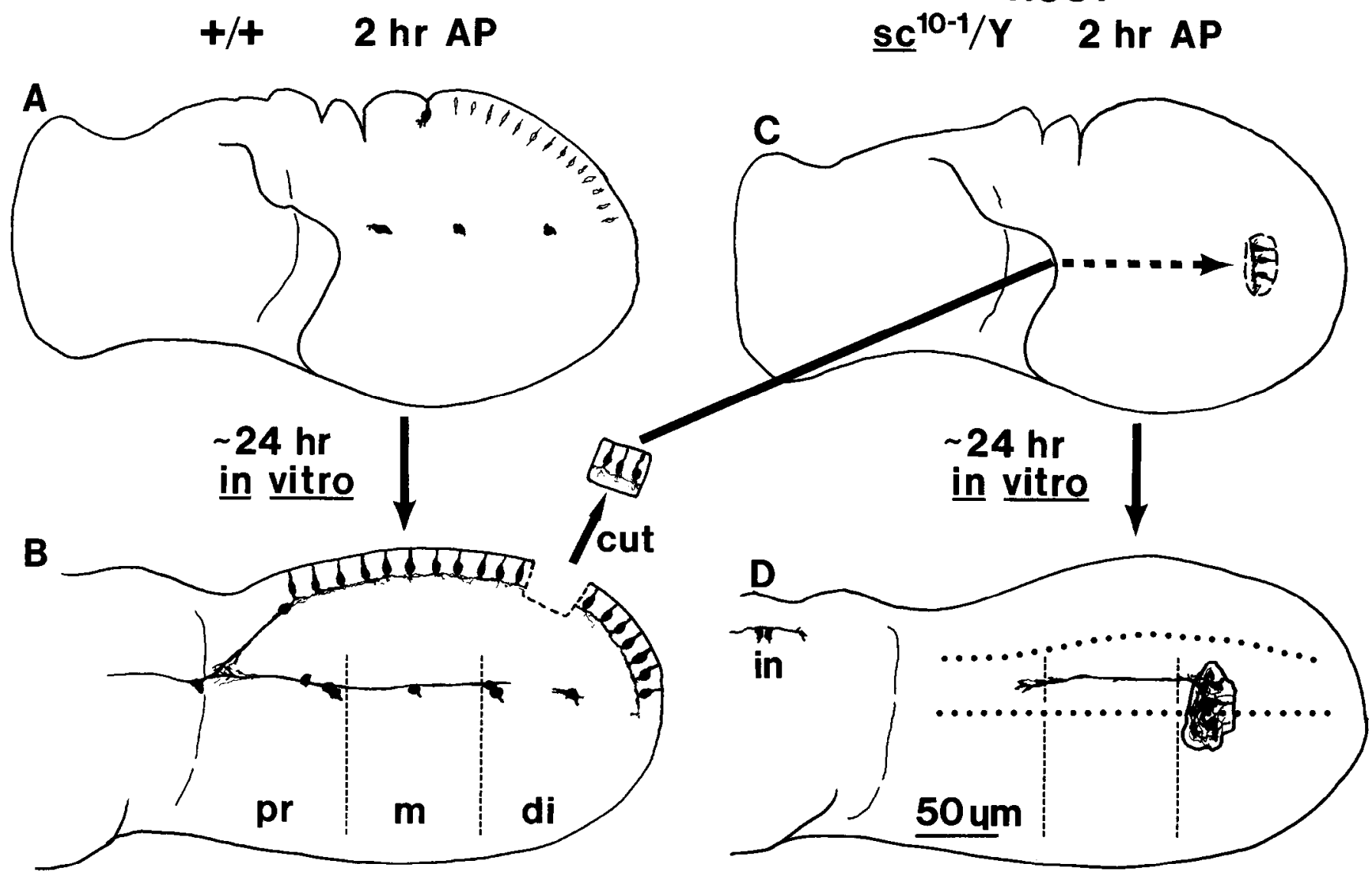

Figure 1. Diagram of transplantation procedure. $A$, Wild-type wing at $2 \mathrm{hr} \mathrm{AP}$, showing positions of neurons. $B$, Wild-type wing reared in vitro from $2 \mathrm{hr}$ AP for $24 \mathrm{hr}$, showing position of the neurons of the bristles and the identifiable campaniform sensilla, and the normal pattern of axon bundles (a second, parallel costal nerve is formed proximally by axons from the costal bristles and campaniform sensilla; this portion of the wing was not analyzed in our experiments and is therefore not shown). Proximal (pr), middle $(m)$, and distal (di) zones are defined for purposes of scoring. An anterior margin fragment is cut out using forceps and a tungsten needle. $C$, scute ${ }^{I 0-1}$ wings at $2 \mathrm{hr}$ AP, showing the lack of intrinsic neurons. The fragment from $B$ is inserted through the opening between the dorsal and ventral epithelia. $D$, Implant-containing host scute wing showing proximal L3 outgrowth of implant neurons and short distal outgrowth from intrinsic neurons in the hinge region (in). Zones marked by thin dashed lines correspond to zones in $B$, used to estimate the position of the implant (Table 1). Proximodistal strip between bold dotted lines corresponds to L3 pathway, defined as region anterior to midline but posterior to anterior quarter of the wing (legend, Table 2).

$\mathrm{AP})$ or along the anterior margin (24 $\mathrm{hr} \mathrm{AP})$ of both in vitro and in vivo wings (Figs. $3 A, 4 A$ ). This debris did not appear in $S c^{I O-1}$ or $f^{36 a}$ (Caltech) wings. Implant axons behaved similarly in both scute stocks.

\section{Results}

Aneural scute wings

In order to test the wing epithelium for the presence of axon guidance cues, neuron-containing fragments were implanted into host wings during the period of normal axonogenesis. Host neurons were excluded as possible guides for axons growing from the implants by using aneural host wings. It has been shown that in male flies carrying the sex-linked $s c^{10-1}$ allele the neurons of the campaniform sensilla of the wing blade never appear, and only a few bristle neurons are formed rather late in development (Schubiger and Palka, 1985; Jan et al., 1985). Similar results were observed when scute wings were removed from pupae at $2 \mathrm{hr}$ AP and reared in vitro (Fig. 2). The neurons of the campaniform sensilla of the wing blade never appeared, even after $48 \mathrm{hr}$ in vitro, and a small number of bristle neurons appeared only some time after $24 \mathrm{hr}$. A few neurons were observed in a cluster near the anterior margin of the hinge region, but while the axons of these intrinsic neurons did sometimes grow distally, they were never observed to extend beyond the most proximal portion of the wing blade. Thus, during at least the first $24 \mathrm{hr}$ of development in vitro, the wing blade proper of the scute wing remained free of intrinsic neurons or their axons.

\section{Wing margin transplantation}

In our initial experiments, neuron-containing donor fragments were obtained from wing anterior margin (Fig. 1). Wild-type wing disks were collected at $2 \mathrm{hr}$ AP and reared for $22-26 \mathrm{hr}$ in vitro. The marginal neurons in such wings are densely clustered and, since at that stage in vitro they are just initiating axonogenesis, provide a good source of growing axons. In vitro reared wings are also relatively free of cuticle (Blair and Palka, 1985a), which facilitates surgical manipulations. Wing margin fragments were transplanted and host wings reared as described in Materials and Methods.

When examined after culturing, the implants could be recognized both by the brightly stained neurons and the increased level of background staining provided by the non-neural implant epithelium. They lay in a variety of positions and orientations 

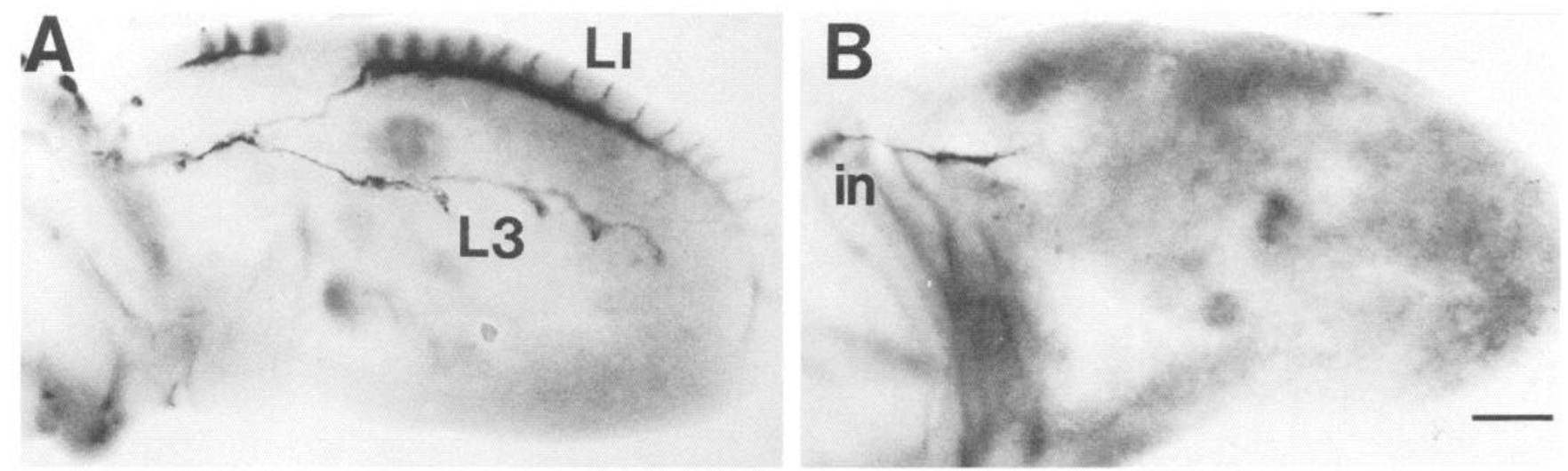

Figure 2. Whole mounts of wings reared in vitro, stained with anti-HRP to visualize neurons. In these and other whole mounts, proximal is to the left and anterior is $u p$. All photographs of whole mounts are negatives of fluorescently labeled wings. $A$, Wild-type wing, removed at 4 hr AP and cultured for $24 \mathrm{hr}$. The sensory neurons formed the 2 nerve bundles of the wing blade, the L1 and L 3 nerves, which join proximally. B, scute ${ }^{\prime O-}$, wing, ren.oved at $2 \mathrm{hr}$ AP and cultured for $24 \mathrm{hr}$. The sensory neurons of the wing blade are lacking, though a cluster of intrinsic neurons (in) remained in the hinge region of the wing and extended axons for a short distance distally. Scale bar, $50 \mu \mathrm{m}$.

within the host wing blade. For the most part, implants did not appear to be incorporated into the host epithelia, but rather lay lodged between them (see Histological analysis below; Fig. 8). In the majority of cases the neurons did not extend axons beyond the limits of donor tissue (Table 1), presumably because the implant had not healed into place sufficiently. However, 136 cases of outgrowth beyond the implant and into the host wing blade yielded the following 2 principal results.

First, we found that axonal outgrowth from the implant was biased in the proximal direction (Table 1). Upon exiting the implant, axons usually joined to form one major axon bundle. In most cases, this bundle had a clear proximal or distal bearing; cases of curved or oblique outgrowth were scored as having a proximal or distal vector component. Part of the clear proximal bias of the entire sample of experimental wings might have been due to the distal position of some implants, which provided little opportunity for distal outgrowth. Therefore, the implants were divided into 3 classes depending upon their position within the wing blade (proximal, middle, or distal, as shown in Fig. $1 B, D$ ). Implants located in the middle of the wing still showed a large bias towards proximal outgrowth, even though they had ample space to extend axons in both directions. Interestingly, almost all the cases of distal outgrowth were from implants located in the proximal-most portion of the wing blade. Proximally located neurons had space to extend axons further proxi- mally, into the hinge and thoracic portions of the wing disk, but nevertheless grew distally in approximately $50 \%$ of the cases (Table 1).

Second, we found that axons usually grew along a band of epithelium whose position corresponded approximately with that of the $\mathrm{L} 3$ nerve bundle in normal wings (compare Fig. $2 A$ with Figs. 3-5, 7). We call this position the L3 pathway (the operational definition used for recognizing this strip is given in Table 2; also see Fig. $1 D$ ). Of 90 implants with outgrowth longer than about $25 \mu \mathrm{m}, 75$ extended axons along or up to the L3 pathway for most of their length (Table 2). Again, while in most cases outgrowth along the L3 path was directed proximally (Figs. $3,4,7), 12$ cases of distal or bipolar L3 outgrowth were observed as well (Figs. $4 B, 5$ ).

The manner in which the axons reached the pathway varied depending upon the position of the implant in the wing blade. The implants were usually fairly large relative to the size of the host wing, so that in most cases they came to lie over or adjacent to the pathway. In almost all such cases the processes exiting the implant did so in the region where it contacted the pathway (Fig. 3, $A, B$; as noted below, this was also true for implants derived from retina, antenna, and leg, Fig. 3, $C-H$ ). This behavior was observed for even the largest wing-derived implants, whose neurons had the opportunity of exiting the implant across a long, non-L3 circumference, demonstrating again the marked

Table 1. Direction of axon outgrowth from implanted neurons

\begin{tabular}{|c|c|c|c|c|c|c|c|c|c|c|c|c|c|}
\hline \multirow[b]{3}{*}{ Donor } & \multicolumn{13}{|c|}{ Position of implant } \\
\hline & \multicolumn{4}{|c|}{ Proximal } & \multicolumn{4}{|c|}{ Middle } & \multicolumn{4}{|c|}{ Distal } & \multirow[b]{2}{*}{ Other } \\
\hline & $\operatorname{Pr}$ & Di & $?$ & 0 & $\operatorname{Pr}$ & Di & $?$ & 0 & $\operatorname{Pr}$ & $\mathrm{Di}$ & $?$ & 0 & \\
\hline Wing margin & 10 & 12 & 10 & 48 & 38 & 1 & 7 & 76 & 52 & 0 & 6 & 59 & 162 \\
\hline Eye disk & 1 & 1 & 2 & 23 & 8 & 3 & 3 & 25 & 8 & 0 & 2 & 23 & 23 \\
\hline Antennal disk & 1 & 0 & 0 & 12 & 2 & 0 & 1 & 8 & 3 & 0 & 0 & 15 & 15 \\
\hline Leg disk & 1 & 4 & 2 & 19 & 12 & 1 & 2 & 36 & 4 & 0 & 1 & 10 & 72 \\
\hline
\end{tabular}

Proximodistal position of the implant estimated as in Figure $1, B$ and $D$. Wings in which the implant was lost or lodged within the hinge region are totaled in "Other." Only the proximal (Pr) or distal (Di) component of the major axon bundle's direction was considered. Purely anteroposteriorly directed outgrowth (growth along creases or anteriorly to L3, see text), as well as irregular or bipolar outgrowth, is included in "?." Cases without growth outside the implant are totaled in " 0 ." 

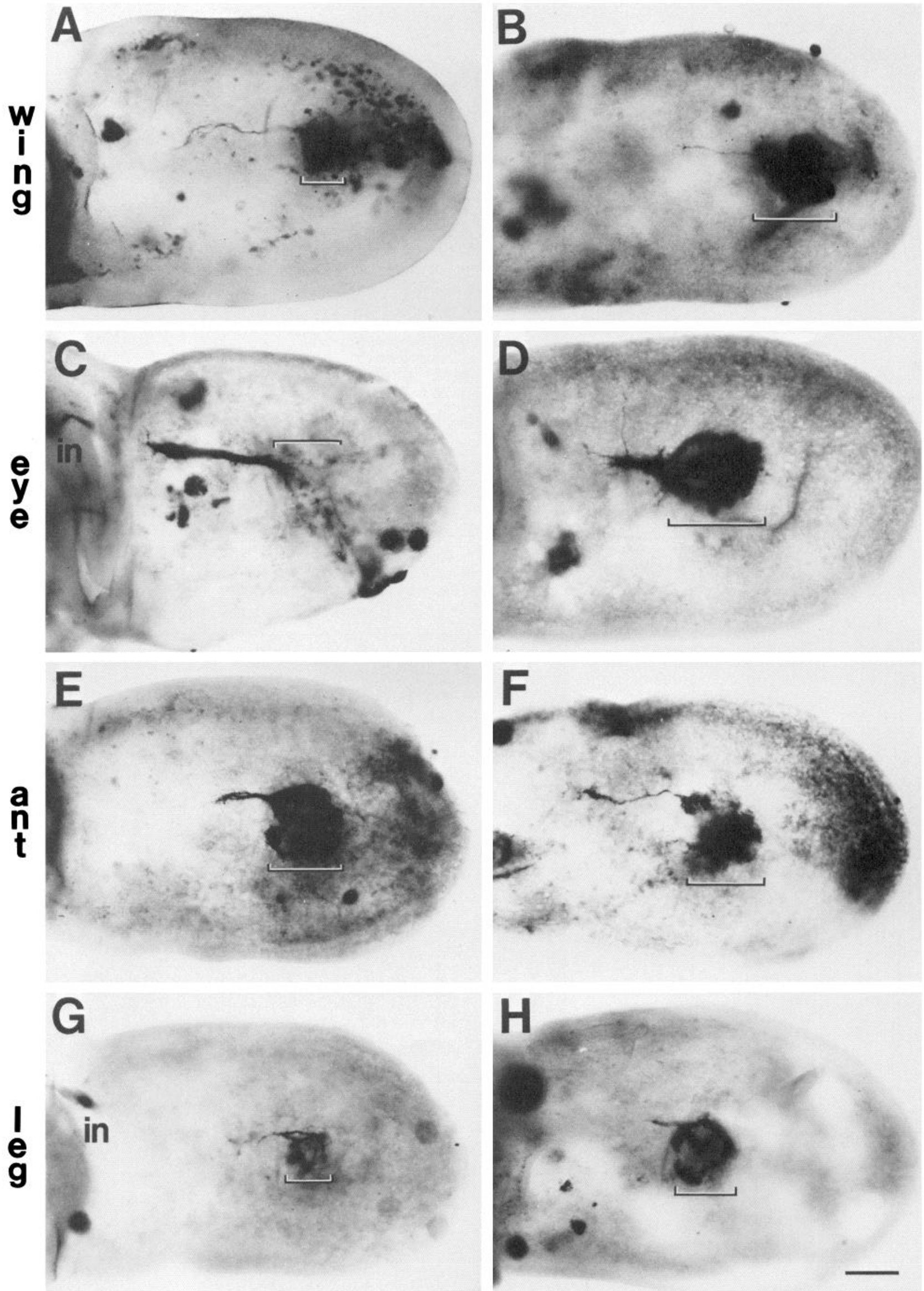

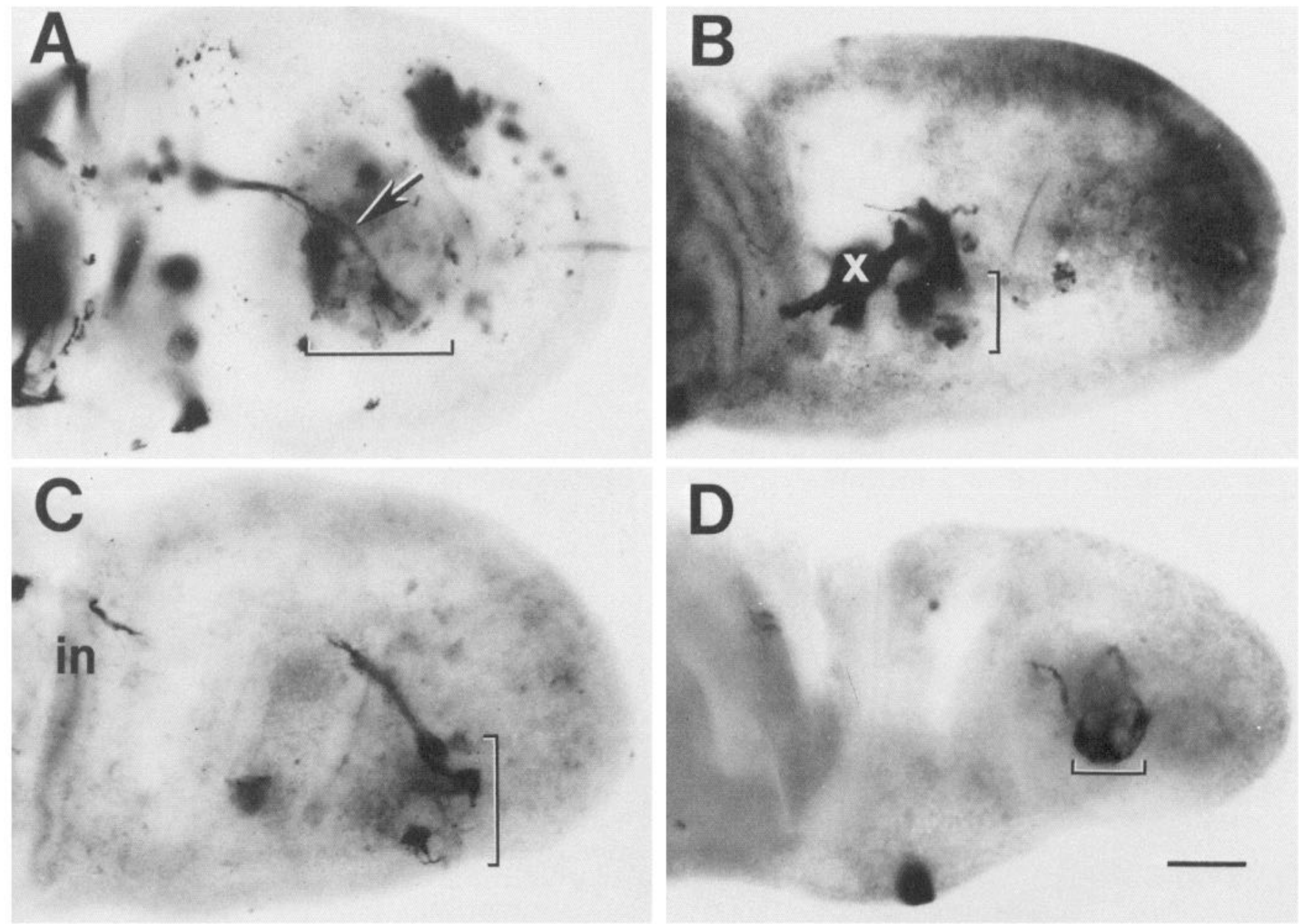

Figure 4. Axon outgrowth directed anteriorly to the $\mathrm{L} 3$ pathway from posteriorly located implants. $A$, Wing implant with antero-proximal outgrowth which curved proximally along L3 pathway. $B$, Wing implant with straight anterior outgrowth which branched to form bipolar process along L3 pathway. The $x$ marks autofluorescent debris. $C$, Leg implant with anteroproximal outgrowth that stopped at L3 pathway. $D$, Leg implant with anteroproximal outgrowth that curved proximally at L3 pathway. in, intrinsic neurons. Scale bar, $50 \mu \mathrm{m}$.

preference for the pathway. Forty-four out of 53 large implants analyzed behaved in this way (only implants that contacted both the L3 pathway and non-L3 regions of the wing and had long axon outgrowth were included in this group).

Outgrowth was not limited, however, to implants in contact with the L3 pathway. If the implant lay posterior to the pathway (Fig. 4), the axons grew anteroproximally (14 cases) or straight anteriorly ( 3 cases) until they reached the pathway; at this point they either stopped (6 cases) or continued to grow along the pathway ( 8 cases). Due to the large size of most implants, none lay anterior to the L3 path without also contacting it.

Only 15 cases of extended non-L3 outgrowth were found, varying in appearance from wing to wing (Fig. 6). This non-L3 outgrowth did not appear to be due to the implant lying in any particular anteroposterior position in the wing. Implants lying completely posterior to the L3 pathway still showed a strong preference for outgrowth towards and along $\mathrm{L} 3$, and the majority of cases of non-L3 outgrowth were from implants in contact with the L3 pathway. The only reproducible type of non-L3 outgrowth observed was that along one or another of the anteroposterior creases in the wing blade, especially the most proximal one. Some implants adjacent to this crease extended axons along it (6 cases), while in other wings axons growing proximally along the L3 path turned upon reaching the crease ( 5 cases, included in "L3-prox" in Table 2; Fig. 6A). Normal neurons, growing in cultured wild-type wings or after different genetic manipulations, sometimes make similar errors in this region (Blair and Palka, 1985a; Blair et al., 1985; Schubiger and Palka, 1985). In 8 of the remaining 9 cases of non-L3 outgrowth, axons either curved (Fig. $6 \mathrm{~B}$ ) or grew at an oblique angle. In only one case did axons "reproduce" the L3 pattern in a non-L3 quadrant of the wing, growing straight proximally through an area posterior to the midline.

The preference for proximal outgrowth along the L3 pathway

Figure 3. Proximal axon outgrowth along the L-3 pathway in scute wings containing implants derived from wing $(A, B)$, eye $(C, D)$, antenna $(E$, $F)$, or leg $(G, H)$ discs. Brackets show the locations of the neuron-containing implants. In all cases the implant lay in contact with the L3 pathway, and axons extended from the implant proximally along this path. Outgrowth was especially dense from eye-derived fragments, and in $D$ appears somewhat diffuse with anteriorly as well as proximally growing processes. Proximal intrinsic neurons (in) were sometimes seen. Scale bar, $50 \mu \mathrm{m}$. 

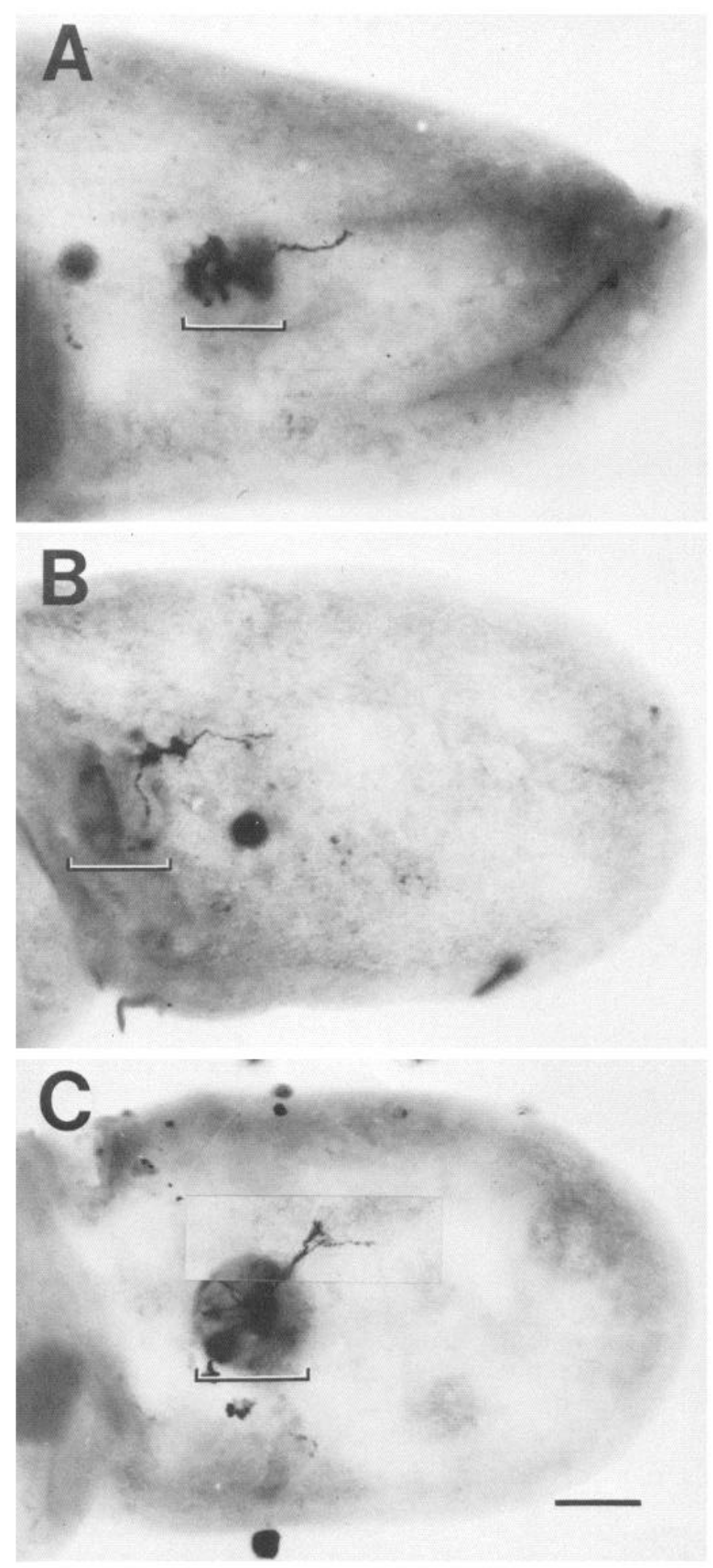

Figure 5. Distal axon outgrowth along L3 pathway from proximally located implants: $A$, Wing implant; $B$, leg implant; $C$, leg implant with short anterodistal outgrowth, which curved straight distally. Scale bar, $50 \mu \mathrm{m}$.

could not be accounted for by the consistent placement or orientation of the transplanted neurons. The implants showed no obvious polarity in host scute wings, usually forming a ball of donor tissue filled with randomly growing axons. In a few cases, processes exited the implants from not just 1 but 2 locations; such axons either joined or grew in parallel trajectories ( 5 cases).

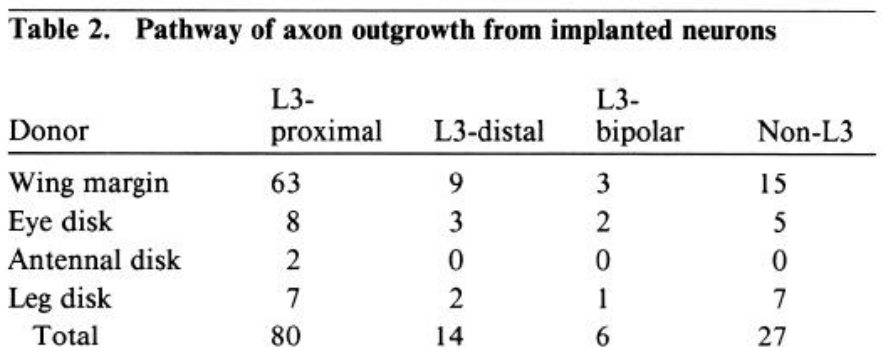

The L3 pathway is judged as a straight strip along the proximodistal axis of the wing, anterior to the midline but posterior to the anterior quarter of the wing (Fig. $1 D$, region between bold dotted lines). Included in the L3-proximal total are 17 cases in which axons grew anteriorly to contact this pathway, and 5 cases in which axons left after growing along it (see text). Two cases in which axons left the L3 path are included in the L3-distal total. Four of the $6 \mathrm{~L} 3$-bipolar cases were posteriorly located implants whose axons grew anteriorly to the L 3 path and there branched in both directions (e.g., Fig. 2D). Cases with less than about $25 \mu \mathrm{m}$ outgrowth were not used for this table.

Furthermore, as stated above, axons exited large implants preferentially, not just in a given direction, but at the specific location of the L3 pathway. And finally, while a few rare implants did lie wholly in the region of the L3 pathway, if only those cases of L3 outgrowth are counted in which the implant was clearly in direct contact with non-L3 regions of the wing (58 cases; not all wings could be analyzed), a comparison with the total number of cases of non-L3 outgrowth (15) still indicates a strong preference for the L 3 path. Therefore, we conclude that cues extrinsic to the implants must be responsible for the guidance of implant axons along the L3 path.

The bias towards outgrowth along the proximodistal axis of the wing cannot be attributed to the insertion of the needle along that axis during the transplantation procedure. The transplantation technique was in some cases modified so that the fragments were inserted directly through the dorsal or ventral epithelia at right angles to the plane of the host wing blade, so that no proximodistal cues were provided (these cases were not included in Tables 1 or 2). Axon outgrowth from such implants was even rarer than that observed using the standard technique, probably because of differences in the healing of the implants into the host wing. However, in all of the 7 cases of long outgrowth observed, the axons showed their usual preference for the L3 pathway (Fig. 7).

\section{Histological analysis}

When developing in vivo, the dorsal and ventral epithelia of the wing come together and the basal processes of their cells interdigitate during the first $8 \mathrm{hr}$ AP. Extended gaps are left, however, between the epithelia, forming a meshwork of channels that run proximodistally along the wing blade; these channels are usually interpreted as the precursors of the adult veins (Waddington, 1940; Anderson, 1984). The L3 nerve grows along the dorsal side of the central-most of these channels, which is therefore considered to correspond to the future L3 (Murray et al., 1984; Blair et al., 1985; Fig. 8, $A, A^{\prime}$ ). These channels are lost when the epithelia separate at $12 \mathrm{hr}$ AP. Mature veins are not formed until the dorsal and ventral epithelia rejoin at 20-24 hr AP (Waddington, 1940; Anderson, 1984).

When viewed as whole mounts, wings reared in vitro for several hours resembled 4-6 hr AP in vivo wings (Blair and Palka, 1985a). It seemed possible that these wings also retained the central pre-vein channel normally present at this stage. If so, this channel could have acted to physically guide the outgrowth 


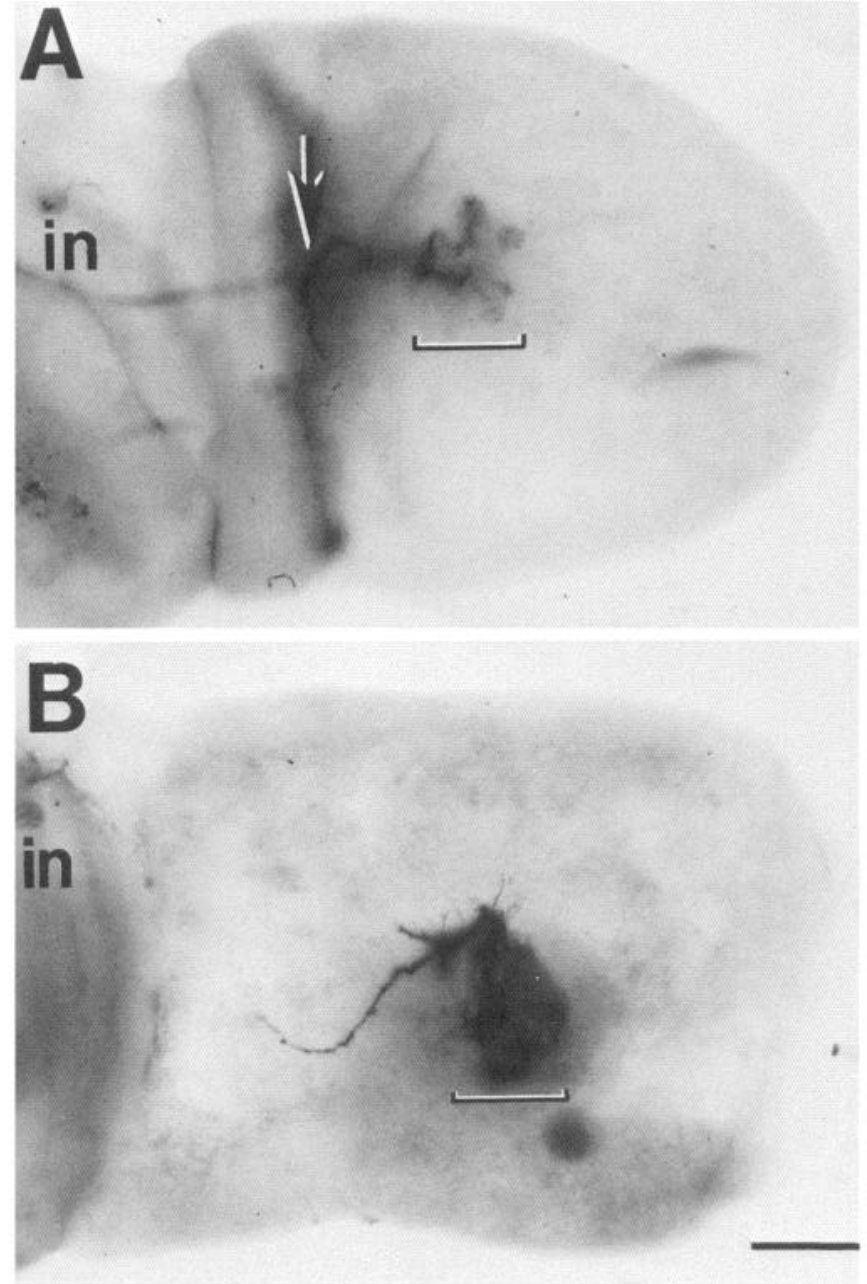

Figure 6. Non-L3 outgrowth. A, Wing implant with proximal L3 outgrowth, which turned posteriorly along a crease in the proximal wing blade (arrow). $B$, Wing implant with proximoposterior outgrowth in the posterior of the wing. in, intrinsic neurons. Scale bar, $50 \mu \mathrm{m}$.

of implant axons along the L3 pathway. Ideally, this possibility could be eliminated by performing transplants on dorsal fragments, but such experiments have proven technically difficult, and as yet have yielded only 3 cases of outgrowth, all of the short L3 type (Blair, unpublished observations). It was therefore important to determine how much of the normal internal histology was preserved in operated wings at the time of implant neuron outgrowth. Because outgrowth beyond the implant did not occur in the majority of implanted wings, its exact timing was difficult to analyze. However, in operated wings that were fixed and observed after $12 \mathrm{hr}$ (removed at $2 \mathrm{hr}$ AP and cultured $10 \mathrm{hr}$ ), only 11 out of 48 cases $(23 \%)$ of outgrowth beyond the implant were observed, as opposed to 136 out of 319 cases $(43 \%)$ for wings reared for $24 \mathrm{hr}$. It is likely, then, that a reasonable percentage of the observed outgrowth along the L3 pathway takes place between 12 and $24 \mathrm{hr}$. Therefore, cross sections of wings growing in situ, wings excised and cultured for 12 and 24 $\mathrm{hr}$, and cultured wings carrying implants were compared.

In sections of cultured, unoperated wings at $8 \mathrm{hr}$-removed at $2 \mathrm{hr}$ AP and cultured $6 \mathrm{hr}$ (Blair et al., 1985)-a nearly normal pattern of channels was present in distal regions but was replaced further proximally by an enlarged central lumen (not shown).

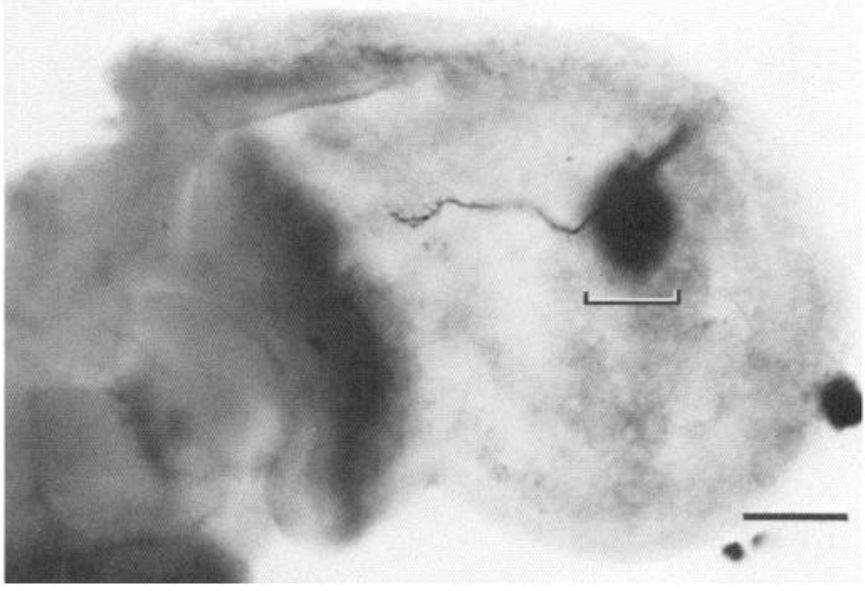

Figure 7. Proximal outgrowth along L3 pathway from wing fragment implanted through a small hole made in the host wing epithelium. Outgrowth also extended anterodistally to the presumed point of insertion (out of focus). Scale bar, $50 \mu \mathrm{m}$.

By $12 \mathrm{hr}$, however, the system of channels was poorly defined in all but the most distal tip of the wing, and normal axons appeared to be growing along the inner surface of an irregular central lumen, interrupted only by sparse contacts between the epithelia (Fig. 8, $B, B^{\prime}$ ). While some vestiges of the central channel were occasionally observed, it was always larger and more poorly defined than the channels seen in $6 \mathrm{hr}$ AP in vivo wings. By $24 \mathrm{hr}$ in vitro the separation of the two epithelia was even more extensive, and in some cases no vestige of the central channel could be found (Fig. 8, $C, C^{\prime}$ ). Similar disruption was observed in $24 \mathrm{hr}$ wings carrying implants. Moreover, in several cases the implant was so large that it appeared to push the host epithelia even further apart (Fig. 8, $D, D^{\prime}$ ). Implant axons in these wings had followed the L3 path; if the wings' morphology had been similar at the time of axon outgrowth, it appears unlikely that such loosely organized channels could direct outgrowth (see Discussion).

\section{Different donors and the lack of specificity}

To test whether the response to the guidance cues in the wing was dependent upon the type of donor neurons used, we repeated the transplantation experiment with tissue derived from $2 \mathrm{hr}$ AP leg or eye-antennal disks (using either the eye or antennal portion of the disk), or $2 \mathrm{hr}$ AP CNS. The proportion of cases of outgrowth from these tissues was lower than that observed using wing margins. Nevertheless, axons from the 3 different types of disk tissue again showed a preference for proximal growth along the L3 pathway (Tables 1 and 2, Fig. 3, $\mathrm{C}-\mathrm{H}$ ). The outgrowth tended to be more diffuse, especially from the eye disk fragments, and the proportion of non-L3 outgrowth was higher than seen using wing margins, especially for leg fragments. However, the number of cases was too small for us to feel confident of these differences. No cases of outgrowth from CNS fragments were observed ( 21 wings total), except in 7 cases of outgrowth to another CNS fragment within the wing.

\section{Discussion}

We performed a series of experiments to examine whether the epithelium of mutant, aneural wings in Drosophila can guide the outgrowth of transplanted, unoriented neurons. From our 

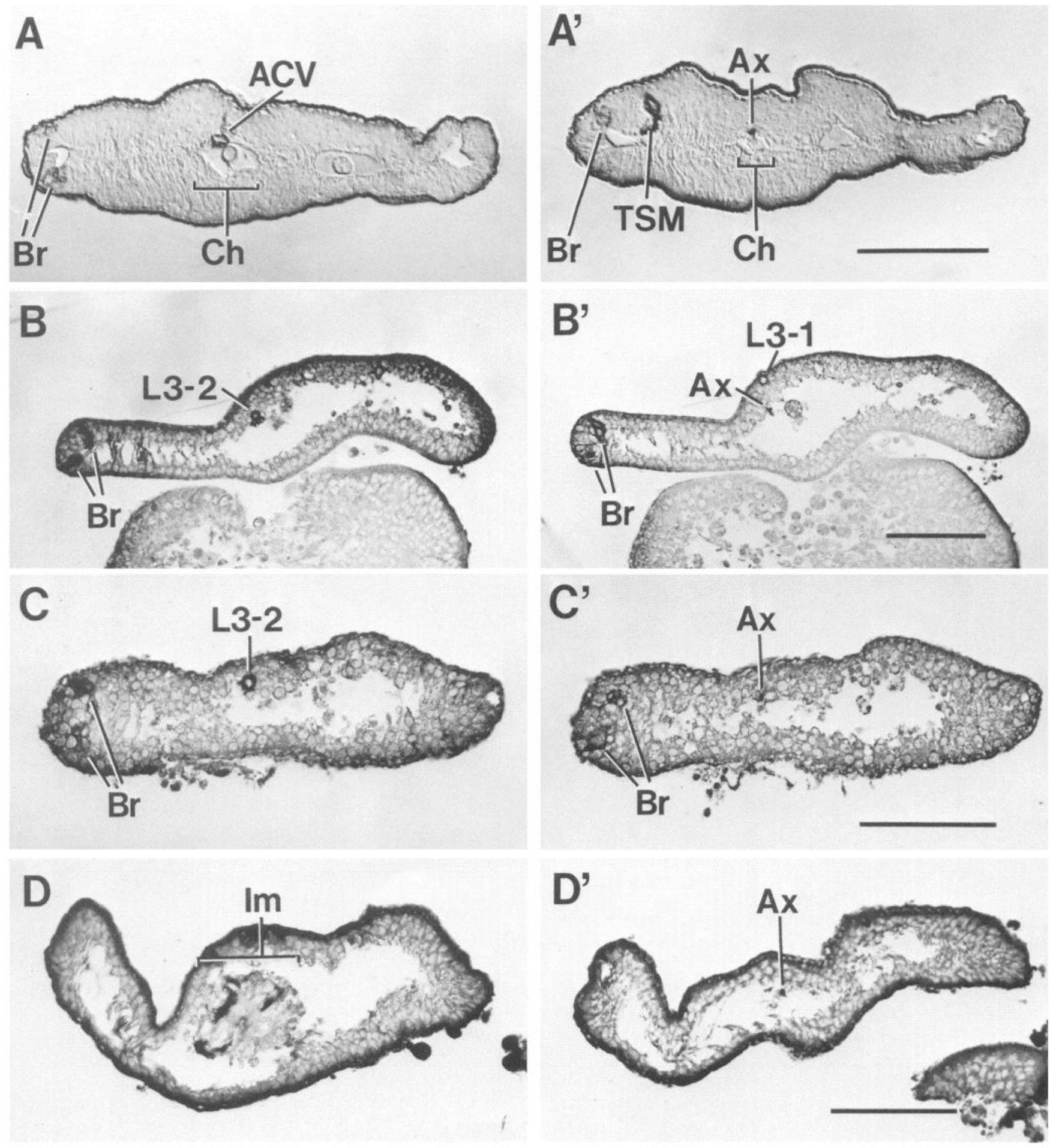

Figure 8. Wing cross sections stained with anti-HRP to visualize neurons. In all sections anterior is to the left and dorsal is $u p$. $A$ and $A^{\prime}$, In vivo wing at $6.5 \mathrm{hr}$ AP. In these 2 sections, DIC optics have been used to increase contrast. $A$, Cell body of the neuron of the anterior cross vein sensillum $(A C V)$, located on the dorsal side of the central channel $(\mathrm{Ch})$. The cell bodies of bristle neurons $(\mathrm{Br})$ are located at the anterior margin. $\mathrm{A}^{\prime}$, A more proximal section containing the axon of the neuron $(A x)$ projecting along the central channel, as well as the cell body of the first twin sensillum neuron (TSM) along the anterior margin. $B$ and $B^{\prime}$, Unoperated in vitro wing at $12 \mathrm{hr}(2 \mathrm{hr}$ AP plus $10 \mathrm{hr}$ in vitro). $B$, Cell body of the neuron of the second dorsal sensillum of the third vein (L3-2) along the large central lumen. The 2 epithelia are only loosely connected. $B^{\prime}$, A more proximal section containing the L3-2 axon projecting along the inner surface of the lumen. This section also contains the neuronal cell body of the first dorsal sensillum of the third vein (L3-1). $C$ and $C^{\prime}$, Unoperated in vitro wing at $24 \mathrm{hr}$ ( $2 \mathrm{hr}$ AP plus $22 \mathrm{hr}$ in vitro). C, Cell body of the L3-2 neuron. $C^{\prime}$, More proximal section containing the L3-2 axon projecting along the very large central lumen. $D$ and $D^{\prime}$, An implanted scute wing at 24 hr. Note the lack of $\mathrm{Br}$ neurons along the anterior margin of the wing. The separation of the dorsal and ventral epithelia of the host wing has apparently been enlarged by the large, neuron-containing implant $(\mathrm{Im})$. $D^{\prime}$, More proximal section containing an axon (or axons) that has grown proximally from the implant over the host epithelium along the L3 pathway. Note the lack of a well-defined central channel. Scale bar, $50 \mu \mathrm{m}$. 
results we draw 2 major conclusions. First, outgrowth from such neurons is biased in the proximal direction, the direction in which normal axons grow in wild-type wings. Second, axons prefer to grow along a band running proximodistally slightly anterior to the wing midline (the "L3 pathway"). This band corresponds approximately to the position occupied in wildtype wings by the nerve of the third vein, 1 of the 2 nerve bundles of the wing blade.

\section{Proximal bias}

Considering only the longitudinal component of the direction of axon outgrowth, a strong bias towards proximal outgrowth was observed. However, this bias was not uniformly distributed throughout the wing blade. Axons growing from implants lodged in distal or middle portions of the wing showed a very strong proximal bias; neurons growing from implants in the proximal portion, in contrast, were about evenly divided between proximal and distal outgrowth (Table 1). Part of this lack of bias might be due to the presence in the proximal wing of a series of transverse creases in the wing blade; these creases seemed particularly attractive to growing axons (see Results) and in some cases may have biased outgrowth from more proximally located implants. However, in several cases axons grew distally past these creases, projecting into the wing blade along the L3 pathway. Therefore, the creases could not have been entirely responsible for the reverse polarity of such outgrowth. Polarity cues would appear to be in some sense weaker or more confusing in the proximal than in the distal wing at the time of implant axonogenesis.

\section{L3 pathway}

Axons from implanted neurons showed a strong tendency to grow along the L3 pathway as defined above (see Table 2). This stereotyped pattern of outgrowth did not appear to be due to the attractiveness of any single point on the wing, but rather the attractiveness of a band of tissue or a series of points along the L3 pathway. Axons exited implants just anterior to the midline, the position of the L3 pathway, and continued growing along the wing at this same anteroposterior position, rather than "homing in" on a particular spot from a variety of locations. In some cases, axons grew both proximally and distally along the pathway (see Fig. $4 B$ ), again as if it were the entire pathway rather than any discrete point that attracted them.

If the implant did not lie in contact with the L 3 pathway but was posterior to it, its axons did not grow at random within the posterior portion of the wing, or proximally as if responding to a distal-to-proximal gradient, even though there appeared to be sufficient space for such outgrowth. Rather, axons grew anteriorly or anteroproximally until they reached the L3 pathway, at which point they either stopped or continued to grow along the path. Thus, the L 3 pathway appears to attract axons at some distance.

The position of the implants could not account for this regularity in axon outgrowth, since large implants contacting both non-L3 and L3 portions of wing, as well as small implants lying distant from L3, all showed a preference for L3 outgrowth. It is possible that large implants might selectively heal to anterior portions of the wing, especially since most implants were derived from the anterior wing margin. However, the L3-bias observed from posteriorly located implants could not be so accounted for; moreover, in spite of a careful search no difference in adhesion between posterior and anterior wing disk cells has yet been observed (Fausto-Sterling and Hsieh, 1987; Fehon et al., 1987).

It is difficult to say just how precisely the observed L3 pathway corresponds to the route of the normal L3 nerve. The host scute wings lack appropriate neuronal landmarks, and cultured wings deform upon mounting, so that the position of the L3 ncrve relative to the wing margins is not exactly reproducible even in wild-type wings. The general impression given by implant axons is that they do not grow as precisely as normal L3 nerves. However, the extent of this imprecision cannot be analyzed without some as yet unavailable positional marker.

\section{The possible role of physical channels}

One possible type of extrinsic guidance cue that might explain the preference for outgrowth along the L3 pathway is the physical channel through which the L3 nerve normally grows (Blair et al., 1985). This channel is formed as a gap between the dorsal and ventral epithelia of the wing, and appears to be the precursor of vein L3 (Waddington, 1940; Anderson, 1984). While this channel can be eliminated from dorsal or ventral fragments of wild-type wings without interfering with the normal pattern of axon outgrowth (Blair et al., 1985), it might act as a physical guide in whole scute wings. However, this appears unlikely for several reasons. First, sections through unoperated and implanted host wings show that the channels in these wings have been severely disrupted, both by the culturing technique and by the presence of the implant, so that the L3 channels is at best very poorly defined (Fig. 8). This open appearance is not due to poor fixation, sincc wings grown in vivo and fixed similarly show defined channels (Fig. $8 \mathrm{~A}$ ); a similar loss of internal structure in cultured wings has been reported by Milner and Muir (1987). Second, the cases in which axons begin posteriorly and grow anteriorly to the L3 position, or grow obliquely through nonL3 portions of the wing, argue that the wing contains few barriers to axon outgrowth, and further that the L3 pathway is attractive over a long distance. Unless the louse meshwork of nonchannel tissue was much more resistant to axon outgrowth than the open space of the channels, and this falling resistance somehow graded towards the channel, it is difficult to see why the purely physical L3 channel should immediately attract axons or filopodia to the exclusion of outgrowth into other parts of the wing. It seems likely, then, that the L3 pathway is made attractive not by its gross physical structure but by the same cues utilized successfully by normal L3 sensory neurons in channel-free dorsal and ventral wing fragments. Purely physical cues cannot, however, be completely ruled out, and may contribute to axon guidance in normal wings.

\section{Other possible guidance mechanisms}

In other insect appendages the evidence supports a proximodistal gradient of substrate-neuron affinity (Nardi, 1983; Caudy and Bentlcy, 1986a). Such a gradient could explain the overall bias towards proximal outgrowth observed in the Drosophila wing. However, the preference for outgrowth along a specific pathway that we observe requires either that affinity be biased not just proximally but also towards L3 (Fig. 9A) or that a second set of cues somehow localizes outgrowth along L3 (Fig. 9, $B$, $C$ ). Since implants located posterior to and out of direct contact with the L3 path do not extend axons at random or straight proximally, but rather grow anteriorly towards L3, it is possible that cues directing outgrowth towards $\mathrm{L} 3$ are found throughout the wing (Fig. 9A). However, an alternative mechanism in which 


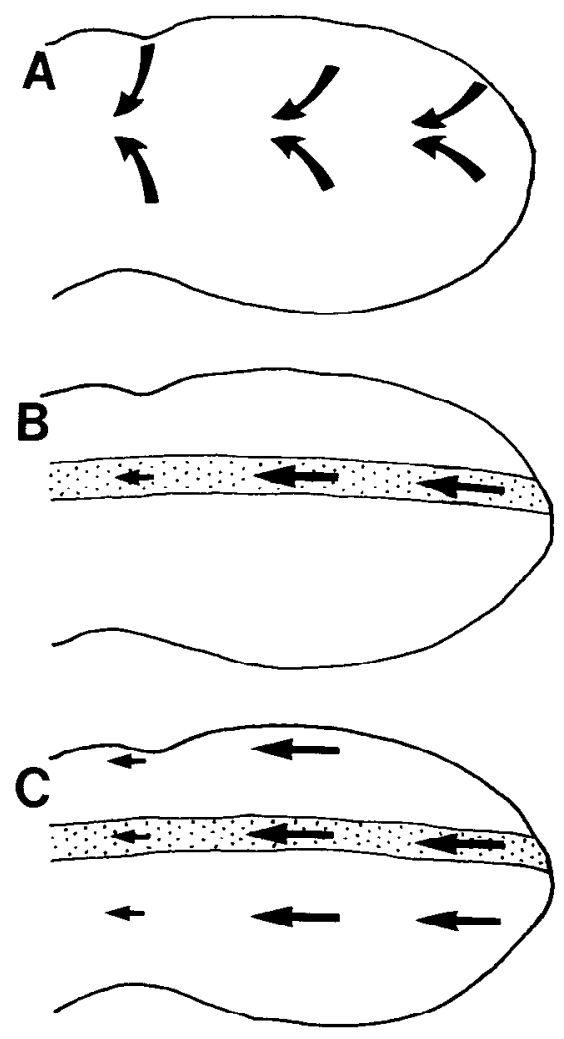

Figure 9. Three possible models of the mechanism of axon guidance in the fly wing. $A$, Single type of guidance cue (arrows), direcling axons throughout the wing towards and proximally along L3. The proximodistal component of the cue becomes weaker in the proximal portion of the wing, allowing some cases of distal outgrowth, but the anteroposterior component remains sufficiently strong to limit outgrowth along L3. Axons might be guided by a gradation of axon affinity within the wing epithelium. $B$, Two types of guidance cues, one favoring outgrowth in either direction along L3 (stippled area) and the other biasing the polarity of outgrowth proximally along L3 (arrows). The polarity cue becomes weaker in the proximal portion of the wing, allowing distal outgrowth along L3. All polarity cues are limited to L3, and axon growth towards $\mathrm{L} 3$ results from random filopodial contact with it. $C$, As $B$, except that the polarity cue is not limited to L3 but is found throughout the wing.

widespread, randomly growing filopodia contact a discrete pathway cannot he ruled out (Fig. 9, $B, C$ ). In the locust leg, the distance at which sensory axons reorient to particular cues, presumably by filopodial contact, is approximately $30-35 \mu \mathrm{m}$ (Caudy and Bentley, 1986b). In our experiments, the implants most distant from the L3 pathway fall within or just outside this range, but it should be noted that filopodia of such length have not been reported in Drosophila.

Finally, the finding that outgrowth along L3 can be directed distally suggests that the proximodistal component of the guidance information is weak enough that it can in some cases be overridden without loss of the L3 component, especially in proximal regions of the wing. Discrete pathways with weak polarity information have also been reported in grasshopper limbs (Bentley and Caudy, 1983b; Caudy and Bentley, 1987) and cultured chick retina (Halfter and Deiss, 1984).

The simplest model that explains the guidance of implant axons in the wing involves the distribution of a single type of adhesive or attractive molecule, graded strongly towards L3 and variably or more weakly in the proximodistal direction (Fig.
$9 A$ ). Such a distributed gradient could also explain the angled, proximoposterior outgrowth of the TSM(1) axon in normal wings, which pioneers the proximal portion of the L1 nerve from the anterior margin to where it joins the L3 nerve (Murray et al., 1984; see Fig. 2A). However, in normal wings the axons of the bristle neurons grow proximally along the marginal $(\mathrm{Ll})$ vein, rather than posteriorward towards vein L3, suggesting that outgrowth in that region is governed by some other set of cues. Implant neurons have never been observed to follow the L1 pathway, indicating that this path may indeed be inaccessible or fundamentally different in character.

\section{Nonspecific highways}

Our results indicate that the $\mathrm{L} 3$ pathway is relatively nonspecific in that it can be followed by neurons not normally found along L3: We have used L1 neurons for most experiments because they are so densely packed (the L3 path is normally occupied by just 7 axons from widely spaced cells), but implants derived from the eye, antenna, or leg disk behave very similarly in the wing (Tables 1 and 2). It is not known whether all or only a subset of neurons from these disks are capable of outgrowth into the wing. A general lack of specificity in pathway selection by sensory axons has been reported before (reviewed in Palka and Ghysen, 1982; Palka, 1986). It contrasts sharply with the specificity of pathway selection within the CNS of insects (Goodman et al., 1984) and is perhaps not surprising since during normal development peripheral sensory neurons do not need to choose between alternate pathways, but rather follow the paths adjacent to their cell bodies (Murray et al., 1984; Jan et al., 1985).

Operationally, the non-neural L3 pathway is much like the guidance "highways" described in chick appendages (Summerbell and Stirling, 1982; Landmesser, 1984; Purves and Lichtman, 1985): It is revealed experimentally by the fact that axons from ectopic neurons can grow towards and along it, and it is relatively nonspecific in that the probing neurons need not be ones that normally utilize the path. Most previous studies on insect appendages have examined only the behavior of normal neurons already located along the usual longitudinal nerve pathways and do not explicitly address the issue of specific nonneural "highways." Thus, the possibility that longitudinal epithelial pathways are found in insect appendages other than the wing remains untested. Leg segment boundaries in grasshoppers can apparently direct axons circumferentially (Bentley and Caudy, 1983b; Caudy and Bentley, 1986a, b, 1987). Such guidance has been attributed to epithelial specializations broadly similar to those we propose for the longitudinally localized L3 pathway.

Since the surface of the wing epithelium along which axons grow can be exposed without a loss of guidance capability (Blair et al., 1985), and since Drosophila is advantageous for both genetic and molecular studies, this would appear to be a favorable experimental system in which to study the general problem of peripheral axon guidance in greater detail.

\section{References}

Anderson, H. (1984) The development of sensory nerves within the wing of Drosophila melanogaster: A light and electron-microscope study. Roux's Arch. 193: 226-233.

Bentley, D., and M. Caudy (1983a) Pioneer axons lose directed growth after selective killing of guidepost cells. Nature 304: 62-65.

Bentley, D., and M. Caudy (1983b) Navigational substrates for periphcral pionccr growth concs: Limb-axis polarity cucs, limb-segment boundaries, and guidepost neurons. Cold Spring Harbor Symp. Quant. Biol. 48: 573-585. 
Bentley, D., and H. Keshishian (1982) Pathfinding by peripheral pioneer neurons in grasshoppers. Science 218: 1082-1088.

Berlot, J., and C. S. Goodman (1984) Guidance of peripheral pioneer neurons in the grasshopper: Adhesive hierarchy of epithelial and neuronal surfaces. Science 223: 493-496.

Blair, S. S., and J. Palka (1985a) Axon guidance in cultured wing discs and disc fragments of Drosophila. Dev. Biol. 108: 411-419.

Blair, S. S., and J. Palka (1985b) Axon guidance in aneural wings of Drosophila. Soc. Neurosci. Abstr. 11: 102.5.

Blair, S. S., M. A. Murray, and J. Palka (1985) Axon guidance in cultured epithelial fragments of the Drosophila wing. Nature 315:406409.

Blair, S. S., M. Schubiger, and J. Palka (1986) Guidance of growing axons through the wing of Drosophila by localized epithelial cues. Soc. Neurosci. Abstr. 12: 56.9 .

Caudy, M., and D. Bentley (1986a) Pioneer growth cone morphologies reveal proximal increases in substrate affinity within leg segments of grasshopper embryos. J. Neurosci. 6: 364-379.

Caudy, M., and D. Bentley (1986b) Pioneer growth cone steering along a series of neuronal and non-neuronal cues of different affinities. $J$. Neurosci. 6: 1781-1795.

Caudy, M., and D. Bentley (1987) Pioneer growth cone behavior at a differentiating limb segment boundary in the grasshopper embryo. Dev. Biol. 119: 454-465.

Fausto-Sterling, A., and L. Hsieh (1987) In vitro culture of Drosophila imaginal disc cells: Aggregation, sorting out, and differentiative abilities. Dev. Biol. 120: 284-293.

Fehon, R. G., A. Gauger, and G. Schubiger (1987) Cellular recognition and adhesion in embryos and imaginal discs of Drosophila melanogaster. In Genetic Regulation of Development, W. F. Loomis, ed., pp. 141-170, Alan R. Liss, New York.

Goodman, C. S., M. J. Bastiani, C. Q. Doe, S. du Lac, S. L. Helfand, J. Y. Kuwada, and J. B. Thomas (1984) Cell recognition during neuronal development. Science 225: 1271-1279.
Halfter, W., and S. Deiss (1984) Axon growth in embryonic chick and quail retinal whole mounts in vitro. Dev. Biol. 102: 344-355.

Ho, R. K., and C. S. Goodman (1982) Peripheral pathways are pioneered by an array of central and peripheral neurones in grasshopper embryos. Nature 297: 404-406.

Jan, Y. N., A. Ghysen, I. Christoph, S. Barbel, and L. Y. Jan (1985) Formation of neuronal pathways in the imaginal discs of Drosophila melanogaster. J. Neurosci. 5: 2453-2464.

Landmesser, L. (1984) The development of specific motor pathways in the chick cmbryo. Trends Neurosci. 7: 336-339.

Milner, M. J., and J. Muir (1987) The cell biology of Drosophila wing metamorphosis in vitro. Roux's Arch. 196: 191-201.

Murray, M. A., M. Schubiger, and J. Palka (1984) Neuron differentiation and axon growth in the developing wing of Drosophila melanogaster. Dev. Biol. 104: 259-273.

Nardi, J. B. (1983) Neuronal pathfinding in developing wings of the moth Manduca sexta. Dev. Biol. 95: 163-174.

Palka, J. (1986) Neurogenesis and axonal pathfinding in invertebrates. Trends Neurosci. 100: 482-485.

Palka, J., and A. Ghysen (1982) Segments, compartments and axon paths in Drosophila. Trends Neurosci. 5: 382-386.

Purves, D., and J. W. Lichtman (1985) Principles of Neural Development, Sinauer Associates, Sunderland, NJ.

Schubiger, M., and J. Palka (1985) Genetic suppression of putative guidepost cells: Effect on establishment of nerve pathways in Drosophila wings. Dev. Biol. 108: 399-410.

Schubiger, M., and J. Palka (1986) Axonal polarity in Drosophila wings with mutant cuticular polarity patterns. Dev. Biol. 113: 461-466.

Shields, G., and J. H. Sang (1977) Improved medium for culture of Drosophila embryonic cells. Dros. Inf. Serv. 52: 161.

Summerbell, D., and R. V. Stirling (1982) Development of the pattern of innervation of the chick limb. Am. Zool. 22: 173-184.

Waddington, C. H. (1940) The genetic control of wing development in Drosophila. J. Genet. 41: 75-137. 